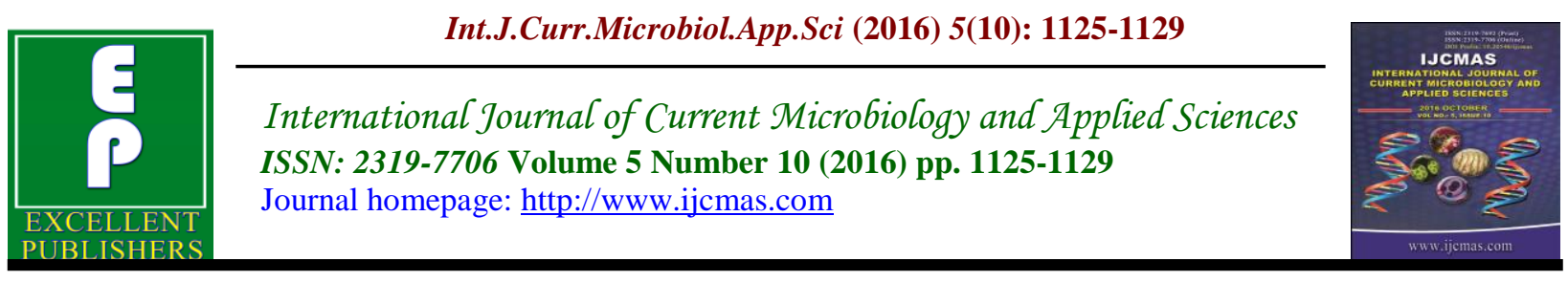

Original Research Article

http://dx.doi.org/10.20546/ijcmas.2016.510.118

\title{
Recognition of Dermatophytes by Dermatophyte Test Medium
}

\author{
Th. Nabakumar Singh ${ }^{1}$, G. Zamzachin ${ }^{2}$ and Ng. Brajachand Singh ${ }^{1}$ \\ ${ }^{1}$ Department of Microbiology, Regional Institute of Medical Sciences (RIMS), \\ Imphal-795001, Manipur, India \\ ${ }^{2}$ Department of Dermatology, Regional Institute of Medical Sciences (RIMS), \\ Imphal-795001, Manipur, India \\ *Corresponding author
}

\begin{abstract}
A B S T R A C T
Keywords

Dermatophytes

/DTM/

SDA/

RIMS

Hospital.

\section{Article Info}

Accepted:

30 September 2016

Available Online:

10 October 2016

The purpose of the study is to determine the clinical variants, their etiological agents and to compare Sabouraud dextrose agar (SDA) and dermatophyte test medium (DTM) for primary isolation of dermatophytes from one hundred clinically suspected cases of dermatophytoses. The culture was done in SDA and DTM and dermatophytes characterization were made by slide culture, urease test and hair perforation test. Among the dermatophytes isolated, the commonest was Trichophyton rubrum (55.22\%) followed by Trichophyton mentagrophytes (34.33\%) and Epidermophyton floccosum (10.45\%). Majority of the patients had tinea corporis $(31.43 \%)$ followed by tinea cruris $(19.40 \%)$. The rate of culture positive by SDA and DTM were $65 \%$ and $67 \%$ respectively which shows no significant difference between the two media for primary isolation of dermatophytes. The present study shows DTM is good for isolation and early recognition of dermatophytes because of a distinct color change in the medium within two weeks time however, standard media such as SDA is required for species identification.
\end{abstract}

\section{Introduction}

In 1969, Taplin and co-workers developed a selective, indicator and differential dermatophyte test medium to provide a simple and rapid method for the detection and presumptive identification of dermatophytes from soldiers with ringworm infections during Vietnam war.

The dermatophytes are a group of keratinophilic molds specialized to grow on the non living keratinized cells of the outer epidermis (stratum corneum), hair and nails of humans and lower animals. Dermatophytosis is a communicable skin disease affecting the skin, hair and nails. There are three closely related genera of dermatophytes : Trichophyton, Microsporum and Epidermophyton. The classic form of this mycosis is ringworm, a circular lesion with an active border, inflammation, pruritus and scaling. Dermatophytosis are among the most widespread and common superficial and cutaneous fungal infections in humans. 
Hence, the present study was undertaken to determine the clinical variants of dermatophytes and their etiological agents and to compare SDA and DTM for their isolation.

\section{Materials and Methods}

This prospective study was carried out in the Department of Microbiology in collaboration with the Department of Dermatology, Regional Institute of Medical Sciences (RIMS), Imphal, Manipur over a period of one year. A total of one hundred specimens were collected from the clinically suspected cases of derrmatophytic skin infections from the patients attending the inpatient and outpatient Department of Dermatology, RIMS, Imphal, Manipur. Samples were collected aseptically and transported in a sterile black paper to avoid exposure to moisture and prevent the growth of contaminants. The samples were screened for the presence of fungal hyphae by $20 \%$ $\mathrm{KOH}$ wet mount examination.

Commercially available DTM was used from TITAN BIOTECH Limited, Bhiwandi, and Rajasthan (TM 083). The ingredients of this media are: soya peptone: $20 \mathrm{~g}$; agar : 10 $\mathrm{g}$; phenol red : $0.29 \mathrm{ml}$; $\mathrm{pH}: 5.5 \pm 2$ at $25^{\circ} \mathrm{C}$, distilled water : 1L. The media was prepared according to manufacturers' guideline \& DTM supplement (chloramphenicol, gentamicin and cyclohexamide) was added. The plates were clear and yellow-orange. SDA with cycloheximide and chloramphenicol was prepared according to the modified method of Eummon's in our media preparation lab. The SDA and DTM were inoculated in duplicate, one incubated at $25^{\circ} \mathrm{C}$ and another at $37^{\circ} \mathrm{C}$ for 3 weeks. SDA was taken as the standard and compared with DTM. The growth of dermatophytes in DTM at $25^{\circ} \mathrm{C}$ was observed as change in the colour of the medium to red within two weeks time but remains yellow in the presence of nonpathogenic fungi. Species identification is more accurately determined with SDA and were confirmed by gross morphology of growth, typical microscopic characteristics, supplemented with slide culture, hair perforation test and urease test.

\section{Results and Discussion}

The results of the findings were tabulated and the data's were analyzed. Out of one hundred samples collected, $57 \%$ were $\mathrm{KOH}$ positive and remaining $43 \%$ were negative. As far as screening in DTM culture media was concerned, 67 of them were positive $(67 \%)$ showing an alkaline (red) reaction on DTM within 2 weeks time; the rest 33 cases were negative as indicated in table 1 . Finally, the screened samples were subjected to culture in SDA media which yielded 65 positive results (65\%). Comparisons of SDA and DTM for the primary isolation of dermatophytes was done as shown in table 2.

The majority of the patients had tinea corporis $(n=21 ; 31.43 \%)$ followed by tinea cruris $(n=13 ; 19.40 \%)$, tinea ungium $(n=8$; $11.94 \%)$, tinea pedis $(n=7 ; 10.44 \%), 6$ cases $(8.96 \%)$ each of tinea manuum and tinea faciei and 3 cases (4.48\%) each of tinea barbae and tinea capitis (Fig.1.). Of the different types of organisms isolated, Trichophyton rubrum (55.22\%) was the predominant pathogen followed by Trichophyton mentagrophytes (34.33\%); Epidermophyton floccosum (10.45\%) as shown in table 3 and fig. 2.

Most of the cases were in the age group of 21-30 years. The least being below 10 years. Males were more commonly affected than females having the ratio being 2.85:1. The maximum number of patients was seen in the months from May to October i.e., during the rainy hot and humid season. 
Dermatophyte infections have been described since ancient time. In the mid 1800s, David Gruby, along with others including Remak, Schoenlein, Lebert, Malmsten and Robin made major contributions to the field. Sabouraud's work, published in the classic Les Teignes, established the current classification in which dermatophytes are classified by genera. In the 1920s, Hopkins and Benkam commenced the actual scientific study of medical mycology. (Rippon JW. In : Medical Mycology : the pathogenic fungi and the pathogenic Actinomycetes $3^{\text {rd }}$ ed. Philadelphia: WB Saunders, 1988). In recent decades, the indiscriminate use of antibiotics, corticosteroids and immunosuppressant have caused an alarming increase in fungal infections.

Dermatophytosis is among the most common dermatological conditions in tropical countries. In the present study, an attempt was made to determine the various clinical patterns of dermatophyte infections and to compare the SDA and DTM for their isolation. Culture is more reliable method of diagnosis than microscopic examination.
The results of culture can be positive even if direct microscopic examination is negative, but is more common for microscopic examination to be positive while culture is negative.

In the present study, the percentage of samples positive by direct microscopy was 57 whereas, the percentage of culture positive was 67 . All the 67 culture +ve cases in DTM produce an alkaline (red) reaction within 14 days time. The red colour change after 14 days must be discarded because saprophytes can induce a similar colour change from this time on. The dermatophytes produce an alkaline (red) reaction on DTM through oxidative deamination, while most of the organisms that are capable of growing on DTM produce an acid reaction (yellow). Approximately, $98 \%$ of fungi producing an alkaline reaction on DTM medium are dermatophytes (Taplin et al., 1969). The present study shows that culture $(67 \%)$ was more sensitive than microscopy $(57 \%)$ in the diagnosis of dermatophytosis. Similar findings were supported by Singh et al, 2003; Sowmya et al, 2014.

Table.1 Isolates showing culture +ve and $-\mathrm{ve} ; \mathrm{KOH}+\mathrm{ve}$ and $-\mathrm{ve}$

\begin{tabular}{|l|c|c|c|}
\hline Culture & Total & KOH+ve & KOH-ve \\
\hline Culture + ve & 67 & 57 & 10 \\
\hline Culture - ve & 33 & 05 & 28 \\
\hline
\end{tabular}

Table.2 Comparision of SDA and DTM in primary isolation of dermatophytes

\begin{tabular}{|c|c|c|}
\hline \multicolumn{2}{|c|}{ Total number of culture positive } & 67 \\
\hline \multicolumn{2}{|c|}{ Culture positive by SDA } & $65(65 \%)$ \\
\hline \multicolumn{2}{|c|}{ Culture positive by DTM } & $67(67 \%)$ \\
\hline SDA & DTM & Total number \\
\hline+ & + & 65 \\
\hline- & + & 02 \\
\hline
\end{tabular}


Table.3 Different species of Dermatophyte isolates

\begin{tabular}{|l|c|c|}
\hline Species & Number & Percentage \\
\hline Trichophyton rubrum & 37 & 55.22 \\
\hline Trichophyton mentagrophytes & 23 & 34.33 \\
\hline Epidermophyton floccosum & 07 & 10.45 \\
\hline
\end{tabular}

Fig.1 Pie diagram showing different types of clinical presentation

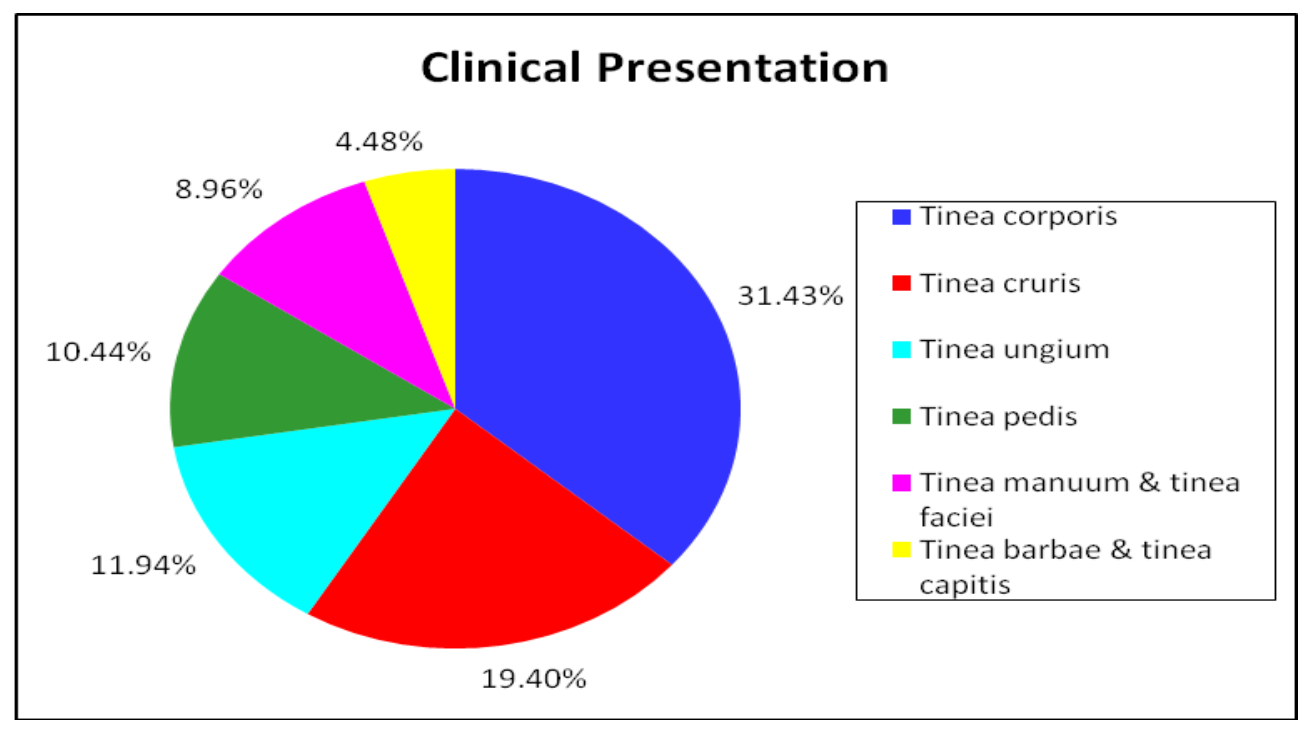

Fig.2 Bar graph showing different fungal isolates

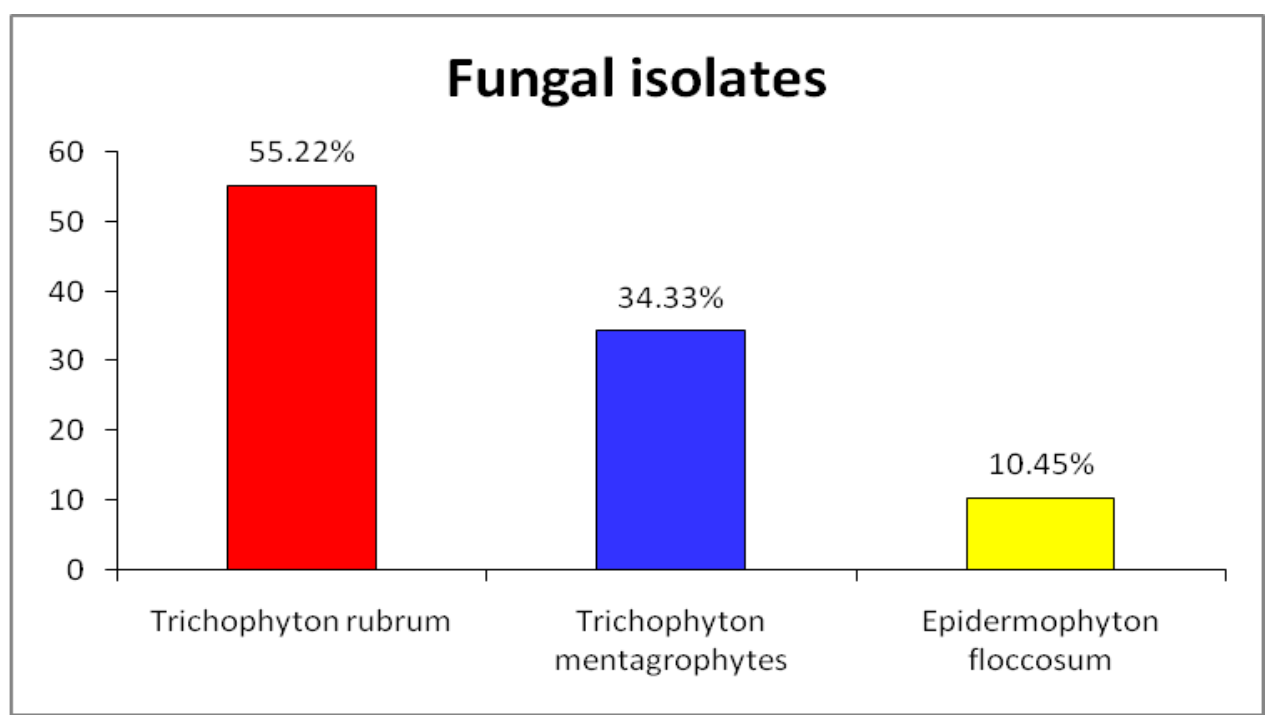

The rates of culture positive by DTM were $(67 \%)$ and by SDA were $(65 \%)$ which shows no significant statistically difference between the two medium for the primary isolation of dermatophytes. Our findings correlated with other workers finding by Singh et al, 2003; Sowmya et al, 2014. 
The present finding also shows that DTM is a good screening medium for the primary isolation and early recognition of dermatophytes from clinical specimens. Species identification is more accurately determined with SDA.

The commonest clinical presentation was tinea corporis $(31.34 \%)$ followed by tinea cruris (19.40\%); tinea ungium (11.94\%). Similar results were observed in the studies of Rani, 1983; Sunderam et al, 1986. Amongst the organisms isolated, Trichophyton rubrum was the most common isolate (55.22\%) followed by Trichophyton mentagrophytes (34.33\%). Similar findings were seen in the studies of Rani,.1983; Varenkar et al.1991.

The commonest age group affected was 21 30 years. The least age group was below 10 years. Similar results were observed by Gujarathi et al, 1996.

Males were commonly affected than females. Similar findings were supported by Gujarathi et al, 1996; Mohanty et al, 1998. Dermatophytosis was seen to occur more in hot and rainy season. Similar results were observed in the studies of Gujarathi et al, 1996. Most of the patients were not complicated. To conclude, DTM is a good screening medium for the selective primary isolation and early detection of dermatophytes from clinical specimens.

\section{References}

Gujarati, U.K., Sivarajan, K., Khubnani, H.,
1996, Dermatomycosis in Loni. Ind J of Med Microbiol. Apr; 14(2): 116-7.

Mohanty, JC., Mohanty, SK., Sahoo, RC., Sahoo, A., Praharaj, CH. 1998. Incidence of Dermatophytosis in Orissa. Ind $\mathbf{J}$ of Med Microbiol. 16 (2): $78-80$

Rani, V. 1983. Study of dermatophyses in Punjabi population. Ind $\mathbf{J}$ Pathol Microbiol. 26:243.

Rippon JW. 1988. The Pathogenic Fungi and the Pathogenic Actinomycetes. In: Medical Mycology: $\quad 3^{\text {rd }}$ ed. Philadelphia : WB Saunders, pp. 140275.

Singh S, Beena PM. 2003. Comparative study of different microscopic techniques and culture media for the isolation of dermatophytes. Ind $\mathbf{J}$ Med Microbiol. 21(1):21-24.

Sowmya N, Appalaraju B. Surendran P, Srinivas CR. 2014. Isolation, Identification and comparative analysis of SDA and DTM for dermatophytes from clinical samples in a tertiary care hospital. IDSRJ of Dental \& Medical Sc. 13(11):68-73.

Sundaram,B.M, et al. 1986. Clinicomycological study of dermatomycoses in Madras (India). Mycosen. 29:230.

Taplin, D., N. Zais, G. Rebel and H. Blank. 1969. Isolation and recognition of dermatophytes on a new medium (DTM). Arch. Dermatol. 99:203-209.

Verenkar M.P. $1991 . \quad$ Clinicomicrobiological study of dermatophytoses. Ind J Pathol Microbiol. 34:186.

\section{How to cite this article:}

Nabakumar Singh, Th., G. Zamzachin and Brajachand Singh, Ng. 2016. Recognition of Dermatophytes by Dermatophyte Test Medium. Int.J.Curr.Microbiol.App.Sci. 5(10): 11251129. doi: http://dx.doi.org/10.20546/ijcmas.2016.510.118 